\title{
Maßgeschneiderte SAP-Therapie
}

_ Trotz vielfältiger Therapien haben Patienten mit stabiler Angina pectoris (SAP) häufig weiterhin Symptome, die ihre Lebensqualität und Aktivitäten beeinträchtigen. Nach Prof. Athanasios Manolis, Athen, sollten daher die aktuellen ESC-Leitlinien kritisch hinterfragt werden. Er bezweifle, ob die Einteilung in First-Line- (z. B. Betablocker) und Second-Line-Therapeutika (z. B. Ranolazin) dem persönlichen Risiko vieler Patienten tatsächlich gerecht werde, zumal alle verfügbaren Antianginosa ungefähr den gleichen Evidenzgrad hätten und keinen nennenswerten Überlebensvorteil zeigten.

\section{Neuer Therapie-Algorithmus}

"Jeder Patient ist anders", betonte Manolis und sprach sich für eine individualisierte Therapie in Abhängigkeit von Hämodynamik und vorliegenden Begleiterkrankungen aus. Er schlug einen Therapie-Al- gorithmus vor, der sich zunächst an Herzfrequenz und Blutdruck des Patienten orientiert [Manolis AJ et al. Int J Cardiology. 2016;220:445-3]. Bei systolischen Druckwerten unter $120 \mathrm{mmHg}$ bevorzugt Manolis hämodynamisch neutrale Medikamente. Bei einer Herzfrequenz unter 60 Schlägen/min sollten keine negtiv chronotropen Medikamente wie z.B. Betablocker eingesetzt werden. Bei systolischen Druckwerten $\geq 120 \mathrm{mmHg}$ oder einer Herzfrequenz $\geq 60$ Schläge/min kämen aber alle Antianginosa in Betracht.

\section{Komorbidität "Diabetes“}

Hier sollten jedoch die jeweiligen Komorbiditäten berücksichtigt werden. So ist z.B. das Piperazinderivat Ranolazin (Ranexå) für AP-Patienten mit Diabetes mellitus eine interessante Option. Denn viele Diabetespatienten leiden nicht nur an makrovaskulären Gefäßläsionen, sondern auch an einer mikrovaskulären An-

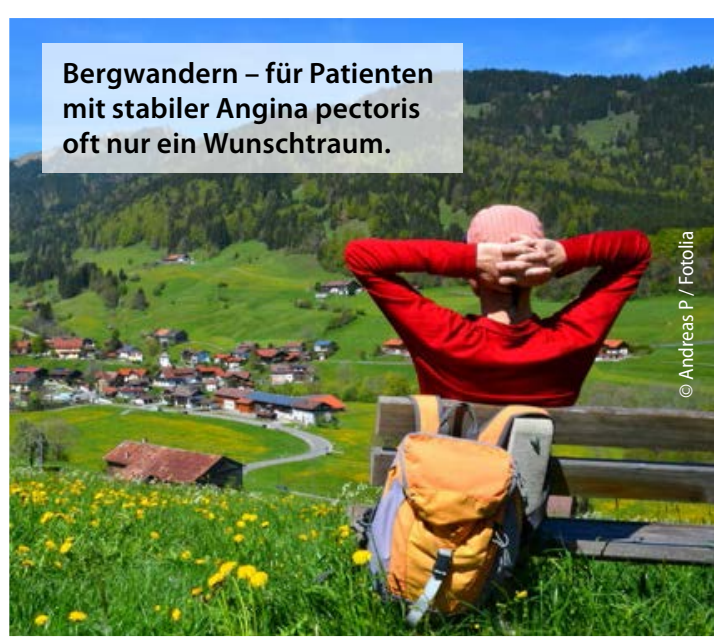

gina. Ranolazin unterbricht als einziges Antianginosum die pathologischen Prozesse der myokardialen Ischämie direkt an der Herzmuskelzelle. Darüber hinaus konnte in Studien eine Senkung des $\mathrm{HbA}_{1 c}$-Wertes um etwa 0,6Prozentpunkte und eine Abnahme des Nüchternblutzuckers beobachtet werden.

\section{Dr. Martina-Jasmin Utzt}

- HerzUpdate 2017; München, Februar 2017 (Veranstalter: Berlin-Chemie)

\section{Asthma bronchiale und COPD}

\section{Extrafeine ICS/LABA-Fixkombination bewährt sich}

_ Bei Asthma und bei COPD kommt es im Krankheitsverlauf zu chronischen Entzündungen und damit zu Umbauprozessen in den kleinen Atemwegen, berichtete Dr. Thomas Voshaar, Moers. Mit der vor zehn Jahren zugelassenen ICS/LABA-Fixkombination aus Beclometason und Formoterol als Dosieraerosol (Foster ${ }^{\otimes}$ ) gelang es mit einer extrafeinen Formulierung, eine gleichmäßige Deposition der Wirkstoffe bis in die Peripherie zu erreichen.

Mit dieser Formulierung habe man in Studien im Vergleich zu nicht-extrafeinen Partikeln mit derselben Wirkstoffkomposition eine bessere Asthmakontrolle, eine stärkere Erhöhung der forcierten Vitalkapazität (FVC), eine stärkere Reduktion der Verschlusskapazität und der bronchialen Hyperreagibilität sowie eine geringere Rate an Exazerbationen erzielt, so Voshaar.

Ähnliches ließ sich für die COPD belegen, für deren Behandlung Foster ${ }^{\circledast}$ im Jahr 2014 die Zulassung erhielt: Es kommt zu einer deutlicheren Zunahme der FVC und der 6-Minuten-Gehstrecke, einer Verbesserung der gesundheitsbezogenen Lebensqualität sowie einer Reduktion der Überblähung und der Exazerbationsrate.

Seit 2013 ist der der TrockenpulverInhalator Foster ${ }^{\circledast}$ Nexthaler ${ }^{\circledast}$ verfügbar. Dieser zeichnet sich durch eine einfache Bedienbarkeit aus. In einer Umfrage beurteilten 74,2\% der Patienten den Nexthaler ${ }^{\circledast}$ als den am einfachsten zu bedienenden Inhalator im Vergleich zu zwei weiteren auf dem Markt befindlichen Inhalatoren mit derselben Wirkstoffkombination. Wie Dr. Rainer Gebhardt, Berlin, berichtete, würden ihn 75,4\% der Patienten bevorzugt einsetzen.

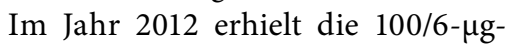
Dosis die Zulassung für das Maintenance-and-Relivery-Therapy(MART)Konzept in der Asthmatherapie.

Auch eine erste extrafeine LABA/ LAMA/ICS-Dreifachkombination zur COPD-Behandlung wurde inzwischen entwickelt und nun bei der Europäischen Arzneimittelagentur zur Zulassung eingereicht.

Veronika Schlimpert

- Jubiläumspressekonferenz „10 Jahre Foster ${ }^{\oplus:}$ Extrafeine Erfolgsgeschichte bis in die kleinen Atemwege"; Hamburg, Oktober 2016 (Veranstalter: Chiesi) 